\title{
Evolution of the leukotoxin promoter in genus Mannheimia
} Jesper Larsen*1, Anders G Pedersen², Robert L Davies³, Peter Kuhnert ${ }^{4}$, Joachim Frey $^{4}$, Henrik Christensen ${ }^{1}$, Magne Bisgaard ${ }^{1}$ and John E Olsen ${ }^{1}$

\begin{abstract}
Address: ${ }^{1}$ Department of Veterinary Pathobiology, Faculty of Life Sciences, University of Copenhagen, Stigbøjlen 4, DK-1870 Frederiksberg C, Denmark, ${ }^{2}$ Center for Biological Sequence Analysis, BioCentrum-DTU, Technical University of Denmark, Building 208, DK-2800 Lyngby, Denmark, ${ }^{3}$ Institute of Biomedical and Life Sciences, Glasgow Biomedical Research Centre, University of Glasgow, 120 University Place, Glasgow G12 8TA, UK and ${ }^{4}$ Institute of Veterinary Bacteriology, University of Berne, Länggass-Strasse 122, CH-3012 Berne, Switzerland
\end{abstract}

Email: Jesper Larsen* - jesl@life.ku.dk; Anders G Pedersen - gorm@cbs.dtu.dk; Robert L Davies - r.l.davies@bio.gla.ac.uk; Peter Kuhnert - peter.kuhnert@vbi.unibe.ch; Joachim Frey - joachim.frey@vbi.unibe.ch; Henrik Christensen - hech@life.ku.dk; Magne Bisgaard - mbi@life.ku.dk; John E Olsen - jeo@life.ku.dk

* Corresponding author

Published: 29 May 2009

BMC Evolutionary Biology 2009, 9:121 doi:10.1।86/147|-2|48-9-121

This article is available from: http://www.biomedcentral.com/l47|-2/48/9//2I

(C) 2009 Larsen et al; licensee BioMed Central Ltd.

This is an Open Access article distributed under the terms of the Creative Commons Attribution License (http://creativecommons.org/licenses/by/2.0), which permits unrestricted use, distribution, and reproduction in any medium, provided the original work is properly cited.
Received: 20 November 2008

Accepted: 29 May 2009

\begin{abstract}
Background: The Mannheimia species encompass a wide variety of bacterial lifestyles, including opportunistic pathogens and commensals of the ruminant respiratory tract, commensals of the ovine rumen, and pathogens of the ruminant integument. Here we present a scenario for the evolution of the leukotoxin promoter among representatives of the five species within genus Mannheimia. We also consider how the evolution of the leukotoxin operon fits with the evolution and maintenance of virulence.
\end{abstract}

Results: The alignment of the intergenic regions upstream of the leukotoxin genes showed significant sequence and positional conservation over a 225-bp stretch immediately proximal to the transcriptional start site of the $l k t C$ gene among all Mannheimia strains. However, in the course of the Mannheimia genome evolution, the acquisition of individual noncoding regions upstream of the conserved promoter region has occurred. The rate of evolution estimated branch by branch suggests that the conserved promoter may be affected to different extents by the types of natural selection that potentially operate in regulatory regions. Tandem repeats upstream of the core promoter were confined to $M$. haemolytica with a strong association between the sequence of the repeat units, the number of repeat units per promoter, and the phylogenetic history of this species.

Conclusion: The mode of evolution of the intergenic regions upstream of the leukotoxin genes appears to be highly dependent on the lifestyle of the bacterium. Transition from avirulence to virulence has occurred at least once in $M$. haemolytica with some evolutionary success of bovine serotype AI/A6 strains. Our analysis suggests that changes in cis-regulatory systems have contributed to the derived virulence phenotype by allowing phase-variable expression of the leukotoxin protein. We propose models for how phase shifting and the associated virulence could facilitate transmission to the nasopharynx of new hosts. 


\section{Background}

The genus Mannheimia includes strains previously classified as trehalose-negative [Pasteurella] haemolytica and is one of the most well-defined and robust clusters within the gamma-proteobacterial family of Pasteurellaceae Pohl 1981 [1]. The Mannheimia species have taken divergent paths toward their distinct lifestyles. The majority of strains isolated from pulmonary infection in cattle belongs to $M$. haemolytica serotype A1/A6 [2]. These strains are sub-dominant to other serotypes (e.g., bovine serotype A2 strains) in the nasopharynx of healthy cattle but dominate when the host defences are at least partly compromised [3-5]. In the immunocompromised host, they have an increased capacity for proliferation and can achieve relatively high total numbers in the nasopharynx, where they are likely to be transmitted to the nasopharynx of new hosts or to spill over or otherwise enter the lungs [6]. However, pulmonary infection caused by M. haemolytica serotype A1 is considered to be non-communicable (i.e. no direct transmission between the lungs) and the continuous circulation of these bacteria in bovine populations seems to depend on their capacity for asymptomatic transmission to the nasopharynx, and not the lungs, of new hosts [7]. By contrast, bovine serotype A2 strains of $M$. haemolytica have had a long history in bovine populations, where they colonise the nasopharynx asymptomatically and rarely cause disease [2]. Also strains of $M$. glucosida serotype A11 and M. ruminalis, the sister group to M. haemolytica $+M$. glucosida, appear to be adapted to a relatively benign lifestyle in the ovine nasopharynx and rumen, respectively $[1,2]$. In addition, bovine strains of M. varigena biogroup 6 , which is the most basal of the Mannheimia species, colonise the nasopharynx asymptomatically, although they have the propensity to cause disease [1]. It therefore seems reasonable to conclude that the genes responsible for the virulence of Mannheimia strains must evolve in response to the demands (selection pressures) associated with a commensal lifestyle rather than any advantages that might arise from causing disease.

Previous works have revealed that the leukotoxin (LktA) protein produced by $M$. haemolytica serotype A1 provides protection from the circulating constitutive and inducible immune defences during pulmonary infection via interactions with host cells [8-10]. Interestingly, much of the virulence can be blamed on the seemingly misguided overresponse of the immune defences [11]. The LktA protein induces the overwhelming activation of neutrophils leading to production of cytokines that mediate tissue injury [12-15].

The leukotoxin (lkt) operon codes for four proteins: an internal acyltransferase, encoded by $l k t C$ [16]; the structural toxin, encoded by $l k t A$, which belongs to the
Escherichia coli HlyA-like subfamily of cytotoxic RTX (repeats in toxin) proteins [16]; an inner membrane protein with a cytoplasmic ATP-binding cassette (ABC) domain, encoded by $l k t B$, which pumps out the LktA protein via interaction with the $C$ terminus of the LktA protein [17]; and a membrane fusion protein, encoded by $l k t D$, which forms a bridge between the inner and outer membranes [17]. The genes for these four proteins are physically adjacent on the chromosome and are transcribed as $l k t C A$ or $l k t C A B D$ messages $[18,19]$.

Recent reports provided the first support for the view that the $l k t$ operon was vertically inherited from the last common ancestor of genus Mannheimia to any ancestor of the diverging species, while changes such as chromosomal rearrangements, pseudogene formation, and deletions of various sizes have occurred in the region upstream of the lkt genes in individual subclades/species [20,21]. However, the extent to which these molecular evolutionary forces affect $l k t$ gene regulation is not known.

In this article, we analysed the lkt promoter among representatives of the five Mannheimia species in order to identify changes in cis-regulatory systems that could have catalysed adaptive evolution. We started our study using multiple sequence alignment as a tool for the identification of orthologous sequences in the intergenic region upstream of the lkt genes (called hereafter the conserved promoter, CP). We also surveyed existing data on regulatory sequences in order to delineate the spatial and functional boundaries of the CP region. On the basis of the multiple sequence alignment, we estimated the rate of evolution for each CP sequence in order to infer the selective constraints. We finally consider how the evolution of the $l k t$ operon fits with the evolution and maintenance of virulence.

\section{Results and discussion}

Table 1 presents the Mannheimia strains analysed in this study, sorted by their overall similarity to $M$. haemolytica serotype A1 str. PHL213. This measure of similarity is based on $16 \mathrm{~S}$ rRNA phylogenies $[1,20]$ and on relationships resolved by multilocus enzyme electrophoresis (MLEE) typing within M. haemolytica + M. glucosida [2].

\section{Conservation and flexibility in the intergenic region upstream of the lkt genes}

The alignment revealed 100\% identity over the entire 406bp intergenic region from $M$. haemolytica serotype A1 str. PHL101 and str. PHL213 (data not shown) and previous reports $[18,22,23]$ allowed us to infer cis-regulatory sequences. The 406-bp region was shared among $M$. haemolytica serotype A2 and M. glucosida serotype A11 as reflected in Figure 1. The alignment showed significant sequence and positional conservation over a 225-bp 
Table I: Strains used in this study

\begin{tabular}{|c|c|c|c|c|c|c|c|}
\hline \multirow[t]{2}{*}{ Subclade ${ }^{a}$} & \multirow[t]{2}{*}{ Taxon ${ }^{b}$} & \multirow[t]{2}{*}{ Strain ID } & \multirow[t]{2}{*}{ Serotype } & \multirow[t]{2}{*}{ Host } & \multirow[t]{2}{*}{ Country } & \multicolumn{2}{|c|}{ GenBank accession no.c } \\
\hline & & & & & & I6S rRNA & Ikt promoter \\
\hline I & Biogroup I & PHL2। 3 & $\mathrm{Al}$ & Bos taurus & & & \\
\hline \multirow[t]{2}{*}{ I } & Biogroup I & CCUG $12392^{\top}$ & $\mathrm{A} 2$ & Ovis aries & UK & [GenBank:AF060699] & [GenBank:AY425276] \\
\hline & M. glucosida & & & & & & \\
\hline \multirow[t]{2}{*}{ I } & Biogroup 3B & $\mathrm{P} 925^{\mathrm{T}}$ & AlI & Ovis aries & Scotland & [GenBank:AF053889] & [GenBank:AY425277] \\
\hline & M. ruminalis & & & & & & \\
\hline \multirow[t]{2}{*}{ II } & Bt 18 biovar 2 & HPAII3 & & Ovis aries & UK & [GenBank:AY425283 & [GenBank:AY425280] \\
\hline & M. granulomatis & & & & & & \\
\hline \multirow[t]{2}{*}{ III } & {$[P .]^{\circ}$ granulomatis } & $\mathrm{PII35/26^{ \top }}$ & & Bos taurus & Brazil & [GenBank:AF053902] & [GenBank:AY425278] \\
\hline & M. varigena & & & & & & \\
\hline IV & Biogroup 6 & $177^{\top}$ & & Bos taurus & Germany & [GenBank:AF053893] & [GenBank:AY425279] \\
\hline
\end{tabular}

a Subclades refer to monophyletic groups based on phylogenetic analysis of I6S rRNA sequences $[1,20]$.

$\mathrm{b} \mathrm{Bt}=$ Bisgaard taxon.

c These sequences have been published elsewhere.

stretch immediately proximal to the transcriptional start site of the $l k t C$ gene among all Mannheimia strains (CP region), with the notable exception of the region corresponding to the repeat tract in M. haemolytica serotype A1 [22], which accommodated many sequence variations (Figures 1 and 2).

In M. haemolytica + M. glucosida, the distal 180-bp stretch of sequence was juxtaposed to the transcriptional start site of the artJ gene (Figure 1). No homology was detected between this 180-bp stretch and the noncoding regions upstream of the CP region in the remaining Mannheimia species (data not shown).

In order to gain some insight into the possible evolutionary mechanisms of this differential pattern, we performed for each species analysis of sequence and positional conservation in the noncoding region upstream of the $\mathrm{CP}$ region. In M. ruminalis and M. varigena, these regions contained stretches of sequence unique to the lapB genes (data not shown). The region of M. granulomatis was adjacent to the $x y l B$ pseudogene and contained a fragment of about $620 \mathrm{bp}$ corresponding to the distal region of the parental $x y l B$ gene as described elsewhere [21]. Moreover, this region contained a 50-bp stretch of inverted sequence that was homologous to the $h s l V$-hslU intergenic regions and stretches of sequence unique to the lapB genes (data not shown).

These observations provide support for the view that the artJ operon, including 180-bp of sequence upstream of the transcribed artJ gene, was translocated into the intergenic region upstream of the lkt genes during early evolu- tion of M. haemolytica + M. glucosida and that the hslvU and lapB operons has undergone pseudogene formation and deletions in M. granulomatis after divergence from the remaining Mannheimia species [21].

These observations illustrate how a few evolutionary events in closely related strains can alter dramatically an intergenic region. In the case of M. haemolytica + M. glucos$i d a$, the alteration coincided with an evolutionary success. Interestingly, transcriptional coupling has been reported for the divergently transcribed artJ and $l k t$ promoters [23]. However, the role of this regulatory feature during transmission of bacteria through host populations is not known. The chimeric promoter was found in all $M$. haemolytica + M. glucosida strains in the present study, irregardless of their propensity to cause disease, suggesting that it evolved in response to selection for something else than making their hosts sick.

\section{Evolutionary rates for $\mathrm{CP}$ sequences}

The role for natural selection in the evolution of regulatory regions is quite unclear [24]. Recognisable features and motifs are often labile, small in size, and may depend on the sequence context $[25,26]$. Therefore, the complex structure of these regions could be due to alternative evolutionary forces [27-30]: (i) compensatory selection occurring when a pair of mutations at different sites that would be singly deleterious produces normal fitness in combination; (ii) stabilising selection could maintain stable levels of gene expression while allowing mutational turnover of functional important sites; (iii) purifying selection could facilitate maintenance of specific functional sites. 

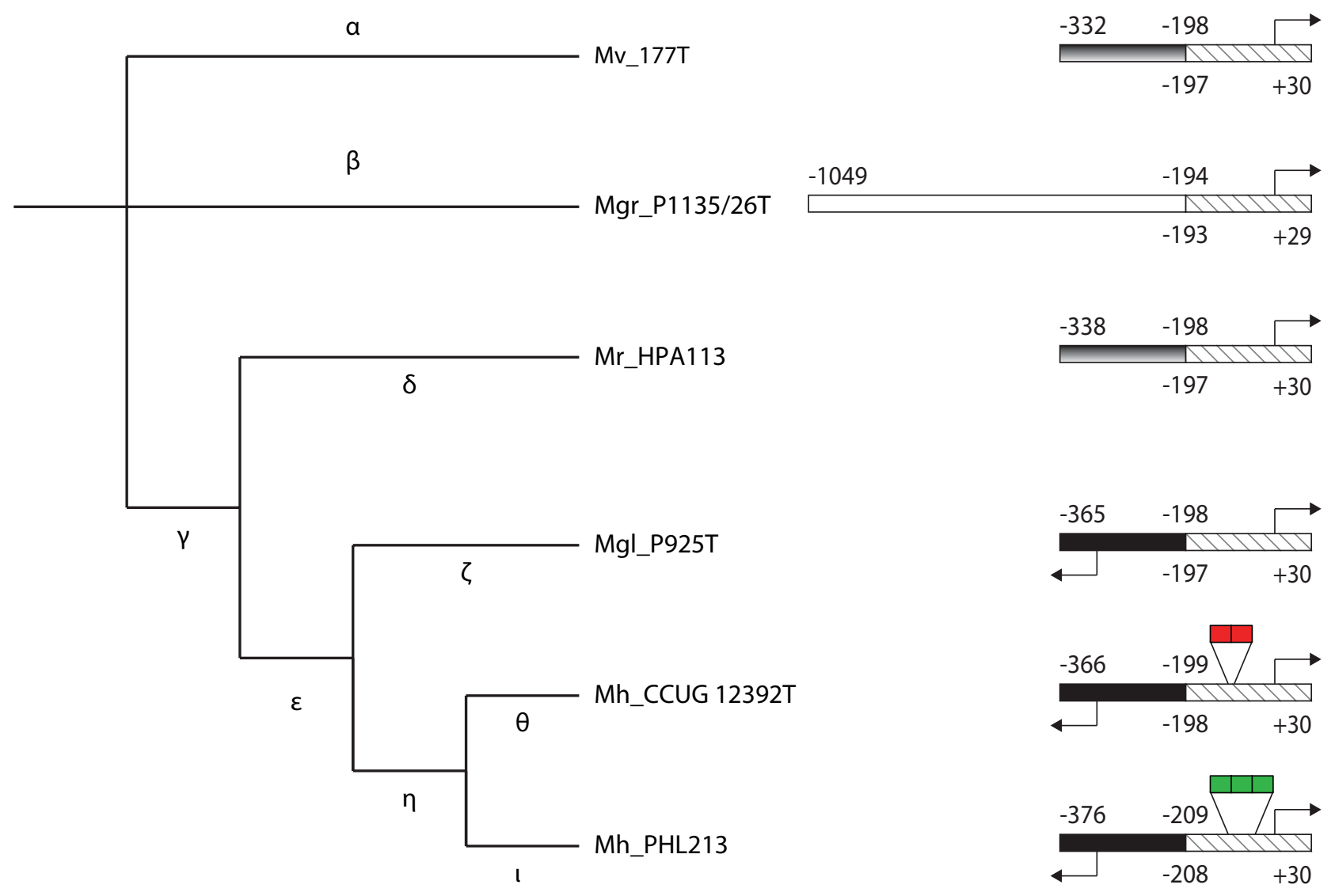

Figure I

Structure and evolution of the intergenic regions upstream of the Ikt genes. Cladogram that describes bifurcation order is based on I6S rRNA phylogenies and MLEE. Greek letters indicate individual branches. The CP region (sequence shared among all Mannheimia strains) is hatched. The site of the repeat sequences in the CP region is indicated by an interruption in hatched box and the number of imperfect and perfect repeat units per promoter is indicated by red and green blocks, respectively. The ancient lapB region is presented by fountain filled box. Individual noncoding regions upstream of the $C P$ region in $M$. haemolytica $+M$. glucosida and $M$. granulomatis are shown by black and white boxes, respectively. Arrows denote the putative transcription start sites. Mh, M. haemolytica: Mgl; M. glucosida; Mr, M. ruminalis; Mgr, M. granulomatis; Mv, M. varigena.

The evolutionary rates for $\mathrm{CP}$ sequences were tested for deviations from expectations derived from the null hypothesis of neutral evolution. The overall length of the 16S rRNA tree (obs. $T_{16 \mathrm{~S}}$ ) was 0.102024 substitutions per site, while the overall length of the CP tree (obs. $T_{\mathrm{CP}}$ ) was 1.33986 substitutions per site. The null hypothesis predicts that the expected length of any given branch on the $\mathrm{CP}$ tree (exp. $v_{\mathrm{CP}}$ ) can be calculated by multiplying the observed length of the corresponding branch on the 16S rRNA tree (obs. $v_{16 S}$ ) by $1.33986 / 0.102024=13.13292$. The observed and the expected lengths for any given branch on the CP tree and their pairwise differences are summarised in Table 2.

For three of four branches ancestral to M. haemolytica serotype A1, the differences between the observed and the expected branch lengths were significantly smaller than zero (the value expected under the null hypothesis of neutral evolution) (Table 2). This is in accord with the operation of stronger levels of purifying selection and possibly reflects an ancient balance between the immune system and these primarily commensal bacteria and their expression of the LktA protein during asymptomatic colonisation of the nasopharynx. The difference between the observed and the expected branch lengths ancestral to $M$. haemolytica $+M$. glucosida was not significantly different from zero (Table 2). This coincided with the translocation of the artJ operon, including 180-bp of sequence upstream of the transcribed art gene, into the intergenic region upstream of the $l k t$ genes [21]. It is possible that this relatively short window of time was dominated by stabilizing selection on this chimeric region, maintaining 
Mh_PHL213

Mh_CCUG $12392 \mathrm{~T}$

$\mathrm{Mg} \bar{l} \mathrm{P} 925 \mathrm{~T}$

Mr $\bar{H} P A 113$

$\mathrm{Mv} 177 \mathrm{~T}$

Mgr_p1135/26T
Mh_PHL213

$\mathrm{Mh}^{-}$CCUG $12392 \mathrm{~T}$

$\mathrm{Mg} \bar{l} \mathrm{P} 925 \mathrm{~T}$

Mr_t

$\mathrm{Mv}{ }^{-} 177 \mathrm{~T}$

Mgr__P1135/26T
1

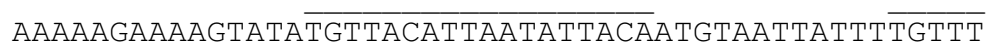
AAAAAGAAAAGTATATGTTACATTAATATTACAATGTAATTATTTTGTTT AAAACAAAAAGTATATGTTACATTAATATTACAATGTAATTATTTTGTTT AAAATGAAAAGTATATGTTACATTGGTTTTACAATGTAATTATTTTGTTT AAAATGAAAAGTAAATATTACATTAATTTTACAATGTAATTATTTCGTTT --AATGATAAATATATGTTACATTAATTTTAGTATGTTACCATTTTGTTT

33444466666777777777777777778888888888889999988888

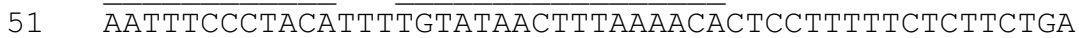

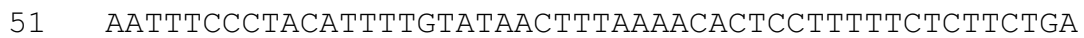
51 AAтTTCCCTACATTTTGTATAACTTTAAAACATTTAATTTTATCTTCTGG 51 AАTTTCCCTACATTTTGTATAACTTTAAAACATTTTATTTTCCAGTCTGT 51 ATTTTCTCTACATTTTGTGTAACTTTAAAGCATTTAATTTTACAACTATT 49 ATTACTAAAACACTTTGTACAAAAAGAAAACATTTTATTTTCCAAATAGT

86777767788888888877777777777644333333333222111111
101

101

101

101

101

99
TTATATAAAAGACAAAAATACAATTTAAGCTACAAAAAACAACAAAAAA
TTATATAAAAGACAAAAAAACAATTAAGCTAAAAAAAACACAAAAAA
CTATACAAAAGACAAAAAATATAATTAAAGCTACAAAAA----------
ACACATAAAAGGCAAAAAATATAATTAAAGCCACAAAAG----------
TTATATAAAAGATAAAAAATTCAACTAAATCCATAAAAA----------
TCATTTAAAA-ATCGAAAATCCAAAAATATGCCAAACAG----------

22223344444444444444334444444333333333100000000000
Mh_PHL213
Mh_CCUG 12392T
Mg]_P925T
Mr_HPA113
Mv_177T
Mgr_P1135/26T
151

151

140

140

140

137

CAACAAAAAACACGACATAAGATCGAGTAATGATTATATTATGTTATAA
t--------ACGACAATAAGATCGAGTAATGATTATATTATGCTATAA
TAACAAAAACACTGCACTTATATCAAAATGTGTTATATTATGCTATAA
TAATAAAAAGTACTGTGGTCTGATCATATAATGAATACATTATGCTATAA
CAATAAAAAACCTGTGGTAAGAGCAGTTGGTTAATGTATTATGCTAAAA
CAGCAAAAAAActggatC-TTTACATAAAAAAGATTATATTATTCTGTC

01111110000111111111111111111222222222222222222222

201

191

190

190

190

186
TTTTTGACCTAATTAGAATAATTATCGAGTGCAAATT
TTTTTGACTAATTAGAATAATATCGAGTGAAATT
TTTTgggTCTAATTAGATAATATCGAGTGCAAAT
TcagcCCCTAATTAGAATAATATCGAGTGCAAAAt
TTTTATTCTAATTAGAATCATTATCGATGTGAAT
TTTCAACTC-AATTCAGAATGATTATCAAGTAAAAACt

22221002555555555555555555555555555533

\section{Figure 2}

Alignment of the CP region. The alignment of the intergenic regions upstream of the lkt genes showed significant sequence and positional conservation over a 225 -bp stretch immediately proximal to the transcriptional start site of the $l k t C$ gene among all Mannheimia strains (CP region), with the notable exception of the region corresponding to the repeat tract in $M$. haemolytica serotype AI [22], which accommodated many sequence variations. Capital letters denote aligned residues. Lower-case letters are not considered to be aligned. Numbers (0-9) below the alignment reflect the relative degree of local similarity among the sequences. Previous works [18,22,23] allowed us to infer cis-regulatory sequences. The footprinted palindromic motifs are indicated by an over line. The repeat sequences are enclosed by a rectangle. The core promoter including the -10 hexamer, the extended TG motifs, and the transcription start site $(+I)$ is highlighted. Mh, M. haemolytica: Mgl; M. glucosida; Mr, M. ruminalis; Mgr, M. granulomatis; Mv, M. varigena. 
Table 2: Evolutionary rate differences for $\mathrm{CP}$ sequences

\begin{tabular}{|c|c|c|c|c|c|c|}
\hline \multirow[t]{2}{*}{ Branch $^{a}$} & \multicolumn{4}{|c|}{ Branch lengths } & \multirow[t]{2}{*}{$p$ value ${ }^{b}$} & \multirow[t]{2}{*}{ Significance $^{c}$} \\
\hline & Obs. $v_{165}$ & Obs. $v_{C P}$ & Exp. $v_{C P}$ & Obs. $v_{C P}-$ Exp. $v_{C P}$ & & \\
\hline$\alpha$ & 0.018827 & 0.247291 & 0.247253 & 0.000038 & 0.992400 & \\
\hline$\beta$ & 0.023416 & 0.720649 & 0.307520 & 0.413129 & 0.002200 & Obs. $v_{C P}>$ Exp. $v_{C P}$ \\
\hline$\gamma$ & 0.015113 & 0.000000 & 0.198478 & -0.198478 & $<0.000100$ & Obs. $v_{C P}<$ Exp. $v_{C P}$ \\
\hline$\delta$ & 0.006960 & 0.117649 & 0.091405 & 0.026244 & 0.490000 & \\
\hline$\varepsilon$ & 0.008202 & 0.074547 & 0.107716 & -0.033169 & 0.434800 & \\
\hline$\zeta$ & 0.003357 & 0.103029 & 0.044087 & 0.058942 & 0.046800 & \\
\hline$\eta$ & 0.015106 & 0.062805 & 0.198386 & $-0.13558 \mid$ & 0.000400 & Obs. $v_{C P}<$ Exp. $v_{C P}$ \\
\hline$\theta$ & 0.002232 & 0.005772 & 0.029313 & $-0.02354 \mid$ & 0.269600 & \\
\hline $\mathrm{l}$ & 0.008811 & 0.008115 & 0.115714 & -0.107599 & $<0.000100$ & Obs. $v_{C P}<$ Exp. $v_{C P}$ \\
\hline
\end{tabular}

a Greek letters indicate individual branches as in Figure I.

b Two-tailed $p$-value.

c Two-tailed $p$-values $<0.0056$ adjusted by the Bonferroni correction for multiple tests were considered significant.

stable levels of $l k t$ (and art) expression and thus the ancient balance between the immune system and bacteria during asymptomatic colonisation of the nasopharynx.

The difference between the observed and the expected branch lengths ancestral to $M$. granulomatis was significantly greater than zero (Table 2). Moreover, the $\mathrm{CP}$ region appeared to be more variable in M. granulomatis, especially in terms of the overall conservation of cis-regulatory sequences (Figure 2). Most notably, there was a high level of sequence degeneracy in the core promoter, which was highly conserved among the other Mannheimia species. Strains belonging to the bovine taxon of M. granulomatis, including M. granulomatis str. P1135/26 , cause severe skin infection (lechiguana) $[1,31,32]$. Interestingly, these strains have never been isolated from healthy cattle, including the nasopharynx, and the disease seems to be acquired by contact with the human botfly (Dermatobia hominis), i.e. there is no direct transmission between cattle or transmission is short-lived [33]. Moreover, comparison of $l k t A$ genes from different taxa within $M$. granulomatis have shown that those from bovine strains are under significantly relaxed selective constraints (J. Larsen, unpublished results). We propose that genetic diversity of the $\mathrm{CP}$ sequence in this strain reflects functional decay due to relaxed selection outside the nasopharynx, enabling mutations to accumulate in the portions of the genome that encode unused functions in the integumentary system. However, it is also possible that positive Darwinian selection for alternative cis-regulatory sequences has acted as a mechanism for coordinating expression of the LktA protein with the nutritional and environmental conditions of the integument. For example, Larsen et al. [20] have shown that the $\beta$-haemolytic phenotype, a marker of the lktA genotype in M. haemolytica [34], is present in this strain, suggesting at least some functional conservation of the lkt operon.

\section{Evolution of repeat sequences}

Tandem repeats of 5'-ACAAAAAACA-3' upstream of the core promoter were first identified in M. haemolytica serotype A1 str. PHL101 [22]. Our preliminary analysis revealed three and two repeat units per promoter in $M$. haemolytica serotype A1 and serotype A2, respectively (Figure 1). In the latter case, the two repeat units varied from the consensus sequence by one nucleotide change each (Figure 2). Because this region accommodated many sequence variations in the remaining Mannheimia species, the unit sequence could not be determined, suggesting that repeat sequences are confined to M. haemolytica.

The relatively recent appearance of three repeat units (at least after the divergence of $M$. haemolytica serotype A1 and serotype A2 as reflected in Figure 1) may provide us with an opportunity to trace the entire evolutionary process back to its origin. Therefore, we examined the number of repeat units per promoter in 28 additional M. haemolytica strains, which represent the diversity within this species based on MLEE, geographic origin, and host association (Table 3). The distribution of the number of repeat units per promoter showed that there were three or more repeat units in 14 out of $16 \mathrm{M}$. haemolytica strains belonging to MLEE lineage A (Table 3). In the two other strains belonging to MLEE lineage $\mathrm{A}$ as well as strains belonging to MLEE lineages $\mathrm{B}(n=7)$ and $\mathrm{C}(n=5)$, there were only two repeat units per promoter (Table 3 ).

The phylogenetic tree of the distal two repeat units from $M$. haemolytica showed a strong association between the sequence of the repeat units, the number of repeat units per promoter, and the phylogenetic history of this species (Figure 3). First, repeat tracts containing three or more repeat units were restricted to MLEE lineage $A$ as described above and belonged to a cluster (I) of perfect repeat units. Second, repeat tracts containing two repeat units were 
Table 3: Distribution of repeat tract polymorphisms in clonally distinct $M$. haemolytica lineages

\begin{tabular}{|c|c|c|c|c|c|c|c|}
\hline Strain ID & Serotype & Host & Country & $\begin{array}{c}\text { MLEE } \\
\text { lineage }^{\mathrm{a}}\end{array}$ & $\begin{array}{l}\text { MLEE } \\
\text { type }^{a}\end{array}$ & $\begin{array}{l}\text { Number of repeat } \\
\text { units per promoter }\end{array}$ & GenBank accession no. \\
\hline $\mathrm{PH} 292$ & $\mathrm{~A} 2$ & Ovis aries & UK & C & 22 & 2 & [GenBank:EU089994] \\
\hline $\mathrm{PH} 278$ & $\mathrm{~A} 2$ & Ovis aries & UK & C & 21 & 2 & [GenBank:EU089992] \\
\hline $\mathrm{PH} 202$ & $\mathrm{~A} 2$ & Bos taurus & UK & $\mathrm{C}$ & 21 & 2 & [GenBank:EU089989] \\
\hline PH598 & $\mathrm{A} 2$ & Ovis aries & UK & C & 20 & 2 & [GenBank:EU090008] \\
\hline PH526 & $\mathrm{A} 2$ & Ovis aries & UK & C & 19 & 2 & [GenBank:EU090004] \\
\hline PHI96 & $\mathrm{A} 2$ & Bos taurus & UK & B & 18 & 2 & [GenBank:EU089988] \\
\hline $\mathrm{PH} 550$ & $\mathrm{~A} 2$ & Bos taurus & Germany & B & 17 & 2 & [GenBank:EU090006] \\
\hline $\mathrm{PH} 494$ & $\mathrm{~A} 2$ & Ovis aries & UK & B & 16 & 2 & [GenBank:EU090003] \\
\hline PH588 & $\mathrm{A} / 3$ & Ovis aries & UK & B & 15 & 2 & [GenBank:EU090007] \\
\hline $\mathrm{PH} 484$ & A7 & Ovis aries & UK & B & 14 & 2 & [GenBank:EU090002] \\
\hline PH396 & A7 & Ovis aries & UK & B & 13 & 2 & [GenBank:EU090000] \\
\hline $\mathrm{PH} 296$ & A7 & Ovis aries & UK & B & 12 & 2 & [GenBank:EU089995] \\
\hline PH706 & Al6 & Ovis aries & UK & A & 11 & 2 & [GenBank:EU090009] \\
\hline $\mathrm{PH} 66$ & $\mathrm{~A} / 4$ & Ovis aries & Ethiopia & $A$ & 10 & 2 & [GenBank:EU089987] \\
\hline $\mathrm{PH} 232$ & A6 & Ovis aries & UK & A & 9 & 3 & [GenBank:EU089990] \\
\hline $\mathrm{PH} 284$ & A6 & Ovis aries & UK & A & 8 & 4 & [GenBank:EU089993] \\
\hline PH398 & Al & Ovis aries & UK & $A$ & 7 & 4 & [GenBank:EU09000I] \\
\hline $\mathrm{PH} 8$ & $\mathrm{Al}$ & Ovis aries & UK & $A$ & 6 & 5 & [GenBank:EU089983] \\
\hline $\mathrm{PH} 238$ & A9 & Ovis aries & UK & $A$ & 5 & 4 & [GenBank:EU089991] \\
\hline PH56 & A8 & Ovis aries & UK & $A$ & 5 & 3 & [GenBank:EU089986] \\
\hline PH50 & A5 & Ovis aries & UK & $A$ & 5 & 4 & [GenBank:EU089985] \\
\hline PH388 & A7 & Ovis aries & UK & $A$ & 4 & 4 & [GenBank:EU089999] \\
\hline $\mathrm{PH} 338$ & A9 & Ovis aries & UK & $A$ & 3 & 3 & [GenBank:EU089996] \\
\hline PH540 & Al & Bos taurus & Germany & A & 2 & 4 & [GenBank:EU090005] \\
\hline PH346 & $\mathrm{A} 12$ & Ovis aries & UK & $A$ & I & 3 & [GenBank:EU089997] \\
\hline $\mathrm{PH} 376$ & A6 & Bos taurus & UK & $A$ & I & 3 & [GenBank:EU089998] \\
\hline $\mathrm{PH} 30$ & $\mathrm{Al}$ & Bos taurus & UK & $A$ & 1 & 4 & [GenBank:EU089984] \\
\hline $\mathrm{PH} 2$ & $\mathrm{Al}$ & Bos taurus & UK & $A$ & I & 3 & [GenBank:EU089982] \\
\hline
\end{tabular}

a Multilocus enzyme electrophoresis (MLEE) lineages and types have been published previously [2].

localised in two clusters (II and III) according to the number of repeat units that varied from the consensus sequence (one and two, respectively) and to the MLEE lineage (B and C, respectively) (Figure 3). These observations are consistent with the generally accepted view that tandem arrays of perfect repeats, such as those present in $M$. haemolytica strains belonging to MLEE lineage $\mathrm{A}$, are hotspots for replication errors, resulting in high rates of expansions/contractions.

\section{Role of repeat sequences}

M. haemolytica, like other usually commensal bacteria, must overcome a variety of environmental hurdles to successfully colonise a host, evade the constitutive and inducible defences of this host, migrate from one within-host habitat to another, and be transmitted to another genetically and immunologically distinct host. Presumably to meet these challenges, some bacteria have highly mutable contingency genes that undergo phenotypic switching (phase shifting) [35].

Although the LktA protein plays a role in evading host immunity in the lungs, it may also function as a nasopha- ryngeal colonization and survival factor. We propose that, in response to the selective pressures associated with the host immune system, expression of the LktA protein is maintained at a baseline level during nasopharyngeal colonisation within individual hosts and asymptomatic nose-to-nose transmission between hosts, while expression is above the baseline level during pulmonary infection.

We further propose that expansions/contractions in the number of perfect repeat units in the lkt promoter are associated with phase-variable expression of the LktA protein. The repeat units contain the characteristic sequences of the so-called A-tracts, which are in phase with the helical repeat (i.e. at 10-11 bp intervals). These upstream Atracts function together as transcriptional enhancers and appear to exhibit inherent DNA curvature $[22,23]$. Although the precise mechanism of action of these bacterial enhancers is still unknown, this theory is at least partly supported by previous empirical works [36-38], which demonstrated a strong, but not necessarily direct, relationship between the number of A-tracts per promoter and the level of promoter strength. 
A

B

\begin{tabular}{|l|l|l|l|l|}
\hline 1 & 2 & 3 & 4 & 5 \\
\hline
\end{tabular}

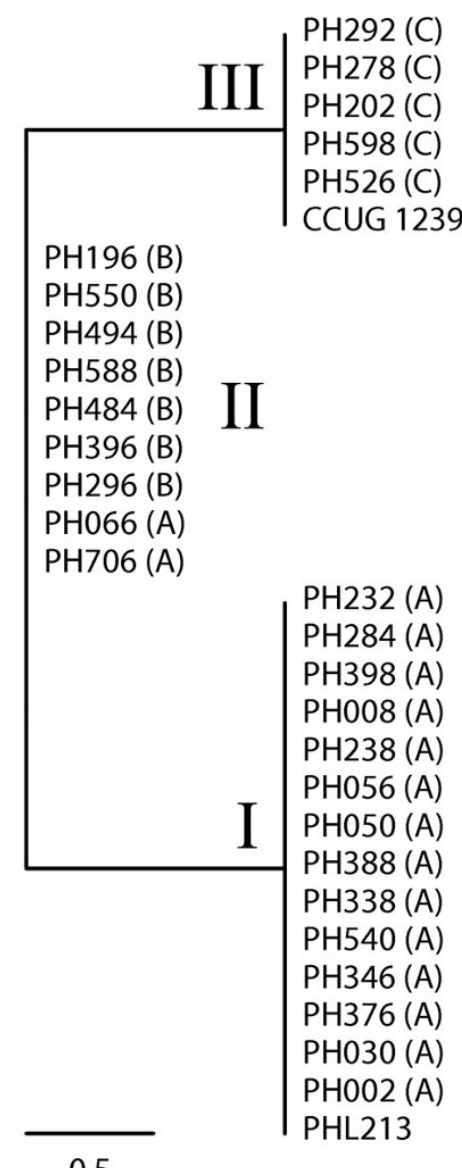

0.5
AAAAAAAACAACAAAAAATA AAAAAAAACAACAAAAAATA

AAAAAAAACAACAAAAAATA

AAAAAAAACAACAAAAAATA

AAAAAAAACAACAAAAAATA

AAAAAAAACAACAAAAAATA

ACAAAAAACAACAAAAAATA

ACAAAAAACAACAAAAAATA

ACAAAAAACAACAAAAAATA

ACAAAAAACAACAAAAAATA

ACAAAAAACAACAAAAAATA

ACAAAAAACAACAAAAAATA

ACAAAAAACAACAAAAAATA

ACAAAAAACAACAAAAAATA

ACAAAAAACAACAAAAAATA

ACAAAAAACAACAAAAAACAACAAAAAACA

ACAAAAAACAACAAAAAACAACAAAAAACAACAAAAAACA

ACAAAAAACAACAAAAAACAACAAAAAACAACAAAAAACA

ACAAAAAACAACAAAAAACAACAAAAAACAACAAAAAACAACAAAAAACA

ACAAAAAACAACAAAAAACAACAAAAAACAACAAAAAACA

ACAAAAAACAACAAAAAACAACAAAAAACA

ACAAAAAACAACAAAAAACAACAAAAAACAACAAAAAACA

ACAAAAAACAACAAAAAACAACAAAAAACAACAAAAAACA

ACAAAAAACAACAAAAAACAACAAAAAACA

ACAAAAAACAACAAAAAACAACAAAAAACAACAAAAAACA

ACAAAAAACAACAAAAAACAACAAAAAACA

ACAAAAAACAACAAAAAACAACAAAAAACA

ACAAAAAACAACAAAAAACAACAAAAAACAACAAAAAACA

ACAAAAAACAACAAAAAACAACAAAAAACA

ACAAAAAACAACAAAAAACAACAAAAAACA

\section{Figure 3}

Evolution of the repeat sequences in M. haemolytica. Maximum-parsimony tree of the two distal repeat units from each promoter (A) and repeat tract polymorphisms (B) using $M$. haemolytica strains, which represent the diversity within this species based on MLEE, geographic origin, and host association. See the text for clusters I, II, and III. The green and red boxes highlight polymorphic sites that match and differ, respectively, from the consensus sequence. The number of repeat units per promoter is indicated. Sequence names contain the strain ID and abbreviations of the corresponding MLEE lineage (A, $B$, or $C)$.

The hypothesis that phase shifting is correlated with pulmonary infection rests on the idea that virulence is a consequence of the within-host evolution for proliferation into new tissues. We tested this hypothesis retrospectively by analysing the frequency distribution of genetic variants among $17 \mathrm{M}$. haemolytica serotype A1 strains isolated from the two within-host habitats (Table 4).

The alignment showed significant sequence and positional conservation over the entire 406-bp stretch among all strains, with the exception that they contained different numbers of repeat units per promoter (data not shown). All strains isolated from the nasopharynx of healthy cattle and representing at least two epidemiological clones based on outer membrane protein (OMP) typing (OMP types 1.2 and 1.4) contained three perfect repeat units per promoter (Table 4). The majority of strains isolated from the lungs of cattle with pulmonary infection and representing at least three epidemiological clones (OMP types $1.1,1.2$, and 1.3 ) also contained three perfect repeat units 
Table 4: Distribution of repeat tract polymorphisms using $M$. haemolytica serotype Al strains collected from the nasopharynx of healthy cattle and the lungs of cattle with pulmonary infection

\begin{tabular}{|c|c|c|c|c|c|c|c|c|}
\hline Strain ID & Country & Farm & $\begin{array}{l}\text { Pulmonary } \\
\text { infection }\end{array}$ & Habitat & $\mathrm{OMPa}$ & LPSa & $\begin{array}{l}\text { Number of repeat } \\
\text { units per promoter }\end{array}$ & GenBank accession no. \\
\hline PHI62 & UK & 1 & Yes & Lungs & I.I & I & 3 & [GenBank: FJ4II257] \\
\hline PHI64 & UK & 2 & Yes & Lungs & 1.2 & I & 3 & [GenBank: F]4||258] \\
\hline PHI66 & UK & 2 & Yes & Lungs & 1.2 & 1 & 3 & [GenBank: F]4II259] \\
\hline $\mathrm{PHI} 68$ & UK & 3 & Yes & Lungs & 1.3 & I & 3 & [GenBank: F]4II260] \\
\hline $\mathrm{PHI} 72$ & UK & 4 & Yes & Lungs & 1.2 & I & 4 & [GenBank: Fj4I|26I] \\
\hline $\mathrm{PHI} 74$ & UK & 4 & Yes & Lungs & 1.2 & I & 4 & [GenBank: F]4II262] \\
\hline $\mathrm{PH} 220$ & UK & $?$ & Yes & Lungs & 1.2 & I & 3 & [GenBank: F]4||263] \\
\hline PHI76 & UK & 5 & No & Nasopharynx & 1.4 & I & 3 & [GenBank: FJ4II264] \\
\hline PHI78 & UK & 5 & No & Nasopharynx & 1.4 & 1 & 3 & [GenBank: F]4||265] \\
\hline PHI80 & UK & 5 & No & Nasopharynx & 1.2 & I & 3 & [GenBank: Fj4II266] \\
\hline PHI82 & UK & 5 & No & Nasopharynx & 1.4 & I & 3 & [GenBank: Fj4II267] \\
\hline $\mathrm{PHI} 84$ & UK & 5 & No & Nasopharynx & 1.2 & I & 3 & [GenBank: F]4||268] \\
\hline PHI86 & UK & 5 & No & Nasopharynx & 1.4 & 1 & 3 & [GenBank: FJ4II269] \\
\hline PHI88 & UK & 5 & No & Nasopharynx & 1.4 & 1 & 3 & [GenBank: F]4| |270] \\
\hline PHI90 & UK & 5 & No & Nasopharynx & 1.4 & 1 & 3 & [GenBank: FJ4I|27I] \\
\hline PHI92 & UK & 5 & No & Nasopharynx & 1.4 & 1 & 3 & [GenBank: F)41|272] \\
\hline PHI94 & UK & 5 & No & Nasopharynx & 1.4 & I & 3 & [GenBank: F]4II273] \\
\hline
\end{tabular}

a Outer membrane protein (OMP) and lipopolysaccharide (LPS) types have been published previously [53].

per promoter, with the exception of two strains (both OMP type 1.2), which contained four perfect repeat units per promoter (Table 4).

The presence of three repeat units per promoter in strains from both within-host habitats suggests that this pattern is associated with a commensal lifestyle in the nasopharynx and that the occasional movement of these bacteria into the lungs may be due to coincidental spill over from the nasopharynx. By contrast, strains containing four repeat units per promoter were confined to the lungs. This observation supports the view that genetic change(s) is (are) required for successful colonisation of the lungs under certain conditions.

Consider an inoculum of $M$. haemolytica that has entered the nasopharynx of a host. Furthermore, assume that successful colonisation of the lungs requires (at least) one change (i.e. addition of one repeat unit) to generate the necessary phenotype (i.e. high-level expression of the LktA protein). Whether or not these mutants exist in the potentially colonising population depends on the total number of cells in the nasopharynx, the fraction of the inoculum that enters the lungs, the rates at which the required genetic change is generated by mutation, and the duration of the carrier state (i.e. the number of generations that survive in the nasopharynx before the population becomes extinct).

This analysis has several potential limitations. First, strain pairs collected from the nasopharynx and lungs of indi- vidual hosts with pulmonary infection (rather than from healthy and sick hosts, respectively) should be used to infer within-host evolution. Second, we were unable to perform in vivo tests of the predictions generated from this analysis because of the lack of a good animal model for $M$. haemolytica.

\section{Selection for phase shifting}

Whether or not virulence associated with phase shifting will be selected at an epidemiological level depends on the trade-off between the transmission advantage accruing to the cell during short bouts of symptoms (sneezing and coughing) and the loss in long-term asymptomatic nose-to-nose transmission due to either increased clearance, rapid host death, or reduced fitness in the nasopharynx, which is the port-of-entry in new hosts.

If symptoms (sneezing and coughing) and transmission are coupled so the more rapid phase shifting (more virulent) bacteria are transmitted at higher rates than the more slow (benign) ones, phase shifting (virulence) would be favoured in the bacterial population. This epidemiological selection model is supported by the apparent evolutionary success of the high virulence $M$. haemolytica serotype A1/A6 [2].

However, if the genetic and phenotypic changes associated with enhanced fitness in the lungs are detrimental to the bacteria back in the nasopharynx, they are unlikely to confer any long-term advantage in their ability to spread from the lungs of one host to the nasopharynx of another 
unless revertant changes occur. In this case, disease is a dead end for these bacteria and is a consequence of shortsighted within-host evolution.

If the asymptomatic and symptomatic sites overlap as in the case of respiratory disease, sneezing and coughing generated by the lung-adapted phenotype could favour transmission of freeriders that remain adapted to the nasopharynx, even when disease is a dead end (i.e. revertant changes do not occur, thus preventing transmission of revertant cells to the nasopharynx of new hosts). In other words, freeriders benefit from the collective action without paying the necessary cost associated with the transmission-enhancing feature.

Finally, we present a second model of the epidemiology and within-host infection dynamics of $M$. haemolytica not considered above. Our association analysis showed that the nucleotide change leading to the origin of short tandem arrays of perfect repeat units coincided with the evolutionary success of $M$. haemolytica strains belonging to MLEE lineage A, including relatively benign ones that colonise the ovine nasopharynx asymptomatically and rarely cause disease. To understand the selective forces responsible for phase shifting in these bacteria, which are not invariably pathogenic, it is important to consider its relevance to the commensal existence of these bacteria at an epidemiological level, as a mechanism to facilitate colonisation of genetically and immunologically diverse hosts.

\section{Conclusion}

The continuous circulation of Mannheimia bacteria in host populations seems to depend on their capacity for asymptomatic nose-to-nose transmission, although other routes of transmission may exist for some species. Our analysis suggests that the origin of short tandem arrays of perfect repeat units have contributed to the derived virulence phenotype of $M$. haemolytica serotype A1/A6 by allowing phase-variable expression of the LktA protein. Further studies are required to better understand the epidemiology of these opportunistic pathogens. Areas of study should include testing the predictions generated from this analysis using both prospective (experimental) and retrospective (epidemiological) methods.

\section{Methods}

\section{Taxa used}

For this study we included M. haemolytica serotype A1 str. PHL213, M. haemolytica serotype A2 str. CCUG 12392T, M. glucosida serotype A11 str. P925T, M. ruminalis str. HPA113, M. granulomatis str. P1135/26 ${ }^{\mathrm{T}}$, and M. varigena str. $177^{\mathrm{T}}$ to balance the number of representatives from the five species within genus Mannheimia (Table 1). These strains have been represented in previous studies by Angen et al. [1,39-41] and Larsen et al. [20,21].

\section{Sequence analysis}

The intergenic regions upstream of the lkt genes were aligned using Dialign 2 [42] with default settings. This segment-based multiple alignment program is known to produce better alignments for the purpose of phylogenetic footprinting than does the tree-based global multiple alignment program ClustalW [43], because it starts by identifying short conserved regions and then incorporates them into a multiple alignment [44].

\section{Estimation of evolutionary rates for CP sequences}

The relationships of the strains in this study were inferred with 16S rRNA; these sequences have been used successfully for systematic studies in genus Mannheimia $[1,20]$. The 16S rRNA sequences were aligned using Dialign 2 [42] with default settings.

Phylogenetic inference from 16S rRNA and CP sequences (Figure 2) was done under the criterion of maximum-likelihood (ML) using PAUP* version 4.0b10 for UNIX [45]. The best fitting model for both data sets was Hasegawa, Kishino, and Yano's [46,47] model with a discrete fourcategory gamma distribution [47] of substitution rate among different nucleotide sites (HKY85 $+\Gamma$ ) based on the Akaike Information Criterion (AIC) computed by the programs Modeltest version 3.06 [48] and PAUP* version 4.0b10 for UNIX [45].

We estimated all HKY85 $+\Gamma$ model parameters (nucleotide frequencies, substitution rates $(\mu)$, branch lengths $(v)$, gamma shape parameter) from the $16 \mathrm{~S}$ rRNA data set. The 16S rRNA tree topology was also found to be the single best estimate when using the program MrBayes version 3.0B4 [49], having a posterior probability (PP) of $100 \%$. The tree was rooted using the $M$. varigena str. $177^{\mathrm{T}}$ sequence as outgroup.

We then used the 16S rRNA tree topology as the basis for determining the ML estimates of the HKY $85+\Gamma$ model parameters from the CP data set, assuming that the 16S rRNA tree topology is a correct representation of the phylogeny connecting the investigated strains. The evolutionary rates for CP sequences were tested for deviation from expectations derived from a neutral evolution model. The null hypothesis is that the $16 \mathrm{~S}$ rRNA and CP sequences have evolved under neutral evolution on the same tree topology but at different rates. If this is the case, we would expect the CP tree to be a scaled version of the 16S rRNA tree (with some noise due to the stochasticity in the substitution process). The null hypothesis predicts the following relationship between the expected length of any given branch on the CP tree and the observed length of the corresponding branch on the $16 \mathrm{~S}$ rRNA tree:

$$
\exp . v_{\mathrm{CP}}=\text { obs. } v_{16 \mathrm{~S}} \times\left(\text { obs. } T_{\mathrm{CP}} / \text { obs. } T_{16 \mathrm{~S}}\right),
$$


where $T$ is the total tree length.

To test whether the observed branch lengths on the $\mathrm{CP}$ tree deviate significantly from what we expect under the null hypothesis of neutral evolution, we performed a socalled parametric bootstrapping analysis [50,51]. The program evolver from the PAML package [52] was used to construct 1,000 synthetic "CP-like" data sets by simulating sequence evolution on the $\mathrm{CP}$ tree and using the HKY85 $+\Gamma$ model parameters estimated from the real CP data set. These synthetic "CP-like" data sets are representatives of CP sequences that have evolved under neutral evolution and they can therefore be used to test whether the observed length of any given branch on the CP tree deviates significantly from what we would expect under the null hypothesis of neutral evolution. We used the program BASEML from the PAML package [52] to perform a ML analysis for each of the 1,000 synthetic "CP-like" data sets using the HKY85 $+\Gamma$ model. For each branch in the 1,000 synthetic "CP-like" trees, we then computed the difference between the observed and the expected branch lengths and tested whether these differences were significantly different from zero (the value expected under the null hypothesis of neutral evolution) by calculating twotailed $p$-values adjusted by the Bonferroni correction for multiple tests (as we have no $a$ priori expectation whether the variation of the difference between the observed and the expected branch lengths should be greater (diversifying evolution) or smaller (unifying evolution) than zero, this test is two-sided).

\section{Phylogenetic analysis of repeat sequences in $\mathbf{M}$. haemolytica}

The number of repeat units per promoter was examined using $28 \mathrm{M}$. haemolytica strains representing the diversity within this species based on MLEE, geographic origin, and host association (Table 3 ) and $17 \mathrm{M}$. haemolytica serotype A1 strains collected from the nasopharynx of healthy cattle and the lungs from cattle with pulmonary infection (Table 4). These strains have been represented in previous studies by Davies et al. [2] and McCluskey et al. [53], respectively. To amplify the intergenic regions in these strains, we designed the forward primer lktpro_UP (5'CCACACACCCGAATAAAAGGGTCAAAAGTG-3') and the reverse primer lktpro_DOWN (5'-GGAGTTCATCCATAGCCAAGTAATGTTTCC-3') from conserved sequences in the 5 ' and 3' flanking genes between the M. haemolytica + M. glucosida strains. The reaction conditions were $2.5 \mathrm{U}$ Taq polymerase, $16 \mathrm{mM}\left(\mathrm{NH}_{4}\right)_{2} \mathrm{SO}_{4}, 67 \mathrm{mM}$ Tris- $\mathrm{HCl}$, $0.01 \%$ Tween-20, $2.5 \mathrm{mM} \mathrm{Mg} \mathrm{SO}_{4}$, each primer at 0.5 $\mathrm{mM}$, and each nucleotide at $0.1 \mathrm{mM}$. The cycling conditions were initial denaturation at $94^{\circ} \mathrm{C}$ followed by 25 cycles of $94^{\circ} \mathrm{C}$ for $30 \mathrm{~s}, 52^{\circ} \mathrm{C}$ for $30 \mathrm{~s}$, and $72^{\circ} \mathrm{C}$ for $30 \mathrm{~s}$, finishing with extension at $72^{\circ} \mathrm{C}$ for $10 \mathrm{~min}$. These PCR products were then directly sequenced.
A phylogenetic tree of the two distal repeat units from 30 M. haemolytica strains (Tables 1 and 3) was reconstructed using the maximum-parsimony method as implemented in the program PAUP* version $4.0 \mathrm{~b} 10$ for UNIX [45]. The first site of the unit sequence was defined arbitrarily by using the AluI restriction site as a landmark [23].

\section{Authors' contributions}

JL carried out the molecular genetic studies, participated in the sequence annotations and in the interpretation of the results, and drafted the manuscript. AGP conducted the phylogenetic analysis of repeat sequences and estimated the evolutionary rates for CP sequences. RLD was responsible for the additional $M$. haemolytica strain collections and participated in the interpretation of the results. PK and JF participated in the design of the study. HC participated in the analysis of gene order data. $\mathrm{MB}$ was responsible for the primary strain collection and participated in the interpretation of the results. JEO conceived of the study, participated in its design and coordination, and helped to draft the manuscript. All authors read and approved the final manuscript.

\section{Acknowledgements}

We thank Christel G Buerholt and Yvonne Schlatter for technical assistance. This work was supported by a grant from the Danish Agricultural and Veterinary Research Council (9702797)

\section{References}

I. Angen $\varnothing$, Mutters R, Caugant DA, Olsen JE, Bisgaard M: Taxonomic relationships of the [Pasteurella] haemolytica complex as evaluated by DNA-DNA hybridizations and I6S rRNA sequencing with proposal of Mannheimia haemolytica gen. nov., comb. nov., Mannheimia granulomatis comb. nov., Mannheimia glucosida sp. nov., Mannheimia ruminalis sp. nov. and Mannheimia varigena sp. nov. Int J Syst Bacteriol 1999, 49(Pt I):67-86.

2. Davies RL, Arkinsaw S, Selander RK: Evolutionary geneticsof Pasteurella haemolytica isolates recovered from cattle and sheep. Infect Immun 1997, 65:3585-3593.

3. Frank GH: Pasteurella haemolytica and respiratory disease in cattle. Proc Annu Meet US Anim Health Assoc 1979, 83:153-I60.

4. Frank GH: When Pasteurella haemolytica colonizes the nasal passages of cattle. Vet Med 1988, 83:1064.

5. Jones CD: Proliferation of Pasteurella haemolytica in the calf respiratory tract after an abrupt change in climate. Res Vet Sci 1987, 42: 179-186.

6. Whiteley LO, Maheswaran SK, Weiss DJ, Ames TR, Kannan MS: Pasteurella haemolytica $A I$ and bovine respiratory disease: pathogenesis. J Vet Intern Med 1992, 6: I I-22.

7. Briggs RE, Frank GH, Purdy CW, Zehr ES, Loan RW: Rapid spread of a unique strain of Pasteurella haemolytica serotype I among transported calves. Am J Vet Res 1998, 59:40I-405.

8. Highlander SK, Fedorova ND, Dusek DM, Panciera R, Alvarez LE, Rinehart C: Inactivation of Pasteurella (Mannheimia) haemolytica leukotoxin causes partial attenuation of virulence in a calf challenge model. Infect Immun 2000, 68:3916-3922.

9. Petras SF, Chidambaram M, Illyes EF, Froshauer S, Weinstock GM, Reese CP: Antigenic and virulence properties of Pasteurella haemolytica leukotoxin mutants. Infect Immun 1995, 63:1033-1039.

10. Tatum FM, Briggs RE, Sreevatsan SS, Zehr ES, Ling HS, Whiteley LO, et al:: Construction of an isogenic leukotoxin deletion mutant of Pasteurella haemolytica serotype I: characterization and virulence. Microb Pathog 1998, 24:37-46. 
II. Ramirez-Romero $\mathrm{R}$, Brogden KA: The potential role of the Arthus and Shwartzman reactions in the pathogenesis of pneumonic pasteurellosis. Inflamm Res 2000, 49:98-I0I.

12. Clarke CR, Lauer AK, Barron SJ, Wyckoff JH III: The role of eicosanoids in the chemotactic response to Pasteurella haemolytica infection. Zentralbl Veterinarmed B 1994, 41:483-49|.

13. Cudd L, Clarke C, Clinkenbeard K: Mannheimia haemolytica leukotoxin-induced increase in leukotriene B4 production by bovine neutrophils is mediated by a sustained and excessive increase in intracellular calcium concentration. FEMS Microbiol Lett 2003, 224:85-90.

4. Radi ZA, Brogden KA, Dixon RA, Gallup JM, Ackermann MR: A selectin inhibitor decreases neutrophil infiltration during acute Mannheimia haemolytica pneumonia. Vet Pathol 2002, 39:697-705.

I5. Slocombe RF, Malark J, Ingersoll R, Derksen FJ, Robinson NE: Importance of neutrophils in the pathogenesis of acute pneumonic pasteurellosis in calves. Am / Vet Res 1985, 46:2253-2258.

16. Lo RY, Strathdee CA, Shewen PE: Nucleotide sequence of the leukotoxin genes of Pasteurella haemolytica AI. Infect Immun 1987, 55:1987-1996.

17. Highlander SK, Chidambaram M, Engler MJ, Weinstock GM: DNA sequence of the Pasteurella haemolytica leukotoxin gene cluster. DNA 1989, 8:15-28.

18. Highlander SK, Engler MJ, Weinstock GM: Secretion and expression of the Pasteurella haemolytica leukotoxin. J Bacteriol 1990 I 72:2343-2350.

19. Strathdee CA, Lo RY: Regulation of expression of the Pasteurella haemolytica leukotoxin determinant. J Bacteriol 1989 | 7 |:5955-5962.

20. Larsen J, Pedersen AG, Christensen H, Bisgaard M, Angen $\varnothing$, Ahrens $P$, et al.: Evidence for vertical inheritance and loss of the leukotoxin operon in genus Mannheimia. J Mol Evol 2007 64:423-437.

21. Larsen J, Kuhnert P, Frey J, Christensen H, Bisgaard M, Olsen JE: Analysis of gene order data supports vertical inheritance of the leukotoxin operon and genome rearrangements in the 5 flanking region in genus Mannheimia. BMC Evol Biol 2007, 7:184

22. Highlander SK, Weinstock GM: Static DNA bending and protein interactions within the Pasteurella haemolytica leukotoxin promoter region: development of an activation model for leukotoxin transcriptional control. DNA Cell Biol 1994 |3:|7|-|8|.

23. Marciel AM, Highlander SK: Use of operon fusions in Mannheimia haemolytica to identify environmental and cis-acting regulators of leukotoxin transcription. Infect Immun 200I, 69:6231-6239

24. Tautz D: Evolution of transcriptional regulation. Curr Opin Genet Dev 2000, 10:575-579.

25. Fessele S, Maier H, Zischek C, Nelson PJ, Werner T: Regulatory context is a crucial part of gene function. Trends Genet 2002 I 8:60-63.

26. Lemon B, Tjian R: Orchestrated response: a symphony of transcription factors for gene control. Genes Dev 2000, | 4:255 |-2569

27. Carter AJ, Wagner GP: Evolution of functionally conserved enhancers can be accelerated in large populations: a population-genetic model. Proc Biol Sci 2002, 269:953-960.

28. Ludwig MZ, Bergman C, Patel NH, Kreitman M: Evidence for stabilizing selection in a eukaryotic enhancer element. Nature 2000, 403:564-567.

29. Ludwig MZ: Functional evolution of noncoding DNA. Curr Opin Genet Dev 2002, I 2:634-639.

30. Stone JR, Wray GA: Rapid evolution of cis-regulatory sequences via local point mutations. Mol Biol Evol 2001, I 8:1764-1770

31. Ribeiro GA, Carter GR, Frederiksen W, Riet-Correa F: Pasteurella haemolytica-like bacterium from a progressive granuloma of cattle in Brazil. J Clin Microbiol 1989, 27: |401-|402.

32. Riet-Correa F, Mendez MC, Schild AL, Ribeiro GA, Almeida SM: Bovine focal proliferative fibrogranulomatous panniculitis (Lechiguana) associated with Pasteurella granulomatis. Vet Pathol 1992, 29:93-103.

33. Ladeira SL, Riet-Correa F, Pereira DB, Carter GR: Role of Pasteurella granulomatis and Dermatobia hominis in the etiology of lechiguana in cattle. Ann N Y Acad Sci 1996, 791:359-368.
34. Murphy GL, Whitworth LC, Clinkenbeard KD, Clinkenbeard PA: Hemolytic activity of the Pasteurella haemolytica leukotoxin. Infect Immun 1995, 63:3209-32I 2.

35. Moxon R, Bayliss C, Hood D: Bacterial contingency loci: the role of simple sequence DNA repeats in bacterial adaptation. Annu Rev Genet 2006, 40:307-333.

36. Aiyar SE, Gourse RL, Ross W: Upstream A-tracts increase bacterial promoter activity through interactions with the RNA polymerase alpha subunit. Proc Natl Acad Sci USA 1998 , 95:14652-|4657.

37. Cheema AK, Choudhury NR, Das HK: A- and T-tract-mediated intrinsic curvature in native DNA between the binding site of the upstream activator NtrC and the nifLA promoter of Klebsiella pneumoniae facilitates transcription. J Bacteriol 1999 , | 8 |:5296-5302.

38. Katayama S, Matsushita O, Jung CM, Minami J, Okabe A: Promoter upstream bent DNA activates the transcription of the Clostridium perfringens phospholipase $\mathbf{C}$ gene in a low temperature-dependent manner. EMBO J 1999, I 8:3442-3450.

39. Angen $\varnothing$, Caugant DA, Olsen JE, Bisgaard M: Genotypic relationships among strains classified under the (Pasteurella) haemolytica-complex as indicated by ribotyping and multilocus enzyme electrophoresis. Zentralbl Bakteriol 1997, 286:333-354.

40. Angen $\varnothing$, Olsen JE, Bisgaard M: Further studies of the relationships among strains classified as taxon 15, taxon 18, taxon 20, (Pasteurella) granulomatis or the (Pasteurella) haemolytica-complex in ruminants using quantitative evaluation of phenotypic data. Zentralbl Bakteriol 1997, 286:3 I7-332.

4I. Angen $\varnothing$, Aalbaek B, Falsen E, Olsen JE, Bisgaard M: Relationships among strains classified with the ruminant Pasteurella haemolytica-complex using quantitative evaluation of phenotypic data. Zentralbl Bakteriol 1997, 285:459-479.

42. Morgenstern B: DIALIGN 2: improvement of the segment-tosegment approach to multiple sequence alignment. Bioinformatics 1999, I5:21|-218.

43. Thompson JD, Higgins DG, Gibson TJ: CLUSTAL W: improving the sensitivity of progressive multiple sequence alignment through sequence weighting, position-specific gap penalties and weight matrix choice. Nucleic Acids Res 1994, 22:4673-4680.

44. Blanchette $M$, Tompa $M$ : Discovery of regulatory elements by a computational method for phylogenetic footprinting. Genome Res 2002, I 2:739-748.

45. Swofford DL: PAUP*: phylogenetic analysis using parsimony (*and other methods), version 4.0b10 Sunderland, Massachusetts, Sinauer Associates; 2003.

46. Hasegawa M, Kishino $H$, Yano $T$ : Dating of the human-ape splitting by a molecular clock of mitochondrial DNA. J Mol Evol $1985,22: 160-174$

47. Yang Z: Estimating the pattern of nucleotide substitution. J Mol Evol 1994, 39: I05-II I.

48. Posada D, Crandall KA: MODELTEST: testing the model of DNA substitution. Bioinformatics 1998, 14:817-818.

49. Ronquist F, Huelsenbeck JP: MrBayes 3: Bayesian phylogenetic inference under mixed models. Bioinformatics 2003, I9:1572-1574.

50. Goldman N: Statistical tests of models of DNA substitution. Mol Evol 1993, 36: 182-198.

51. Huelsenbeck JP, Hillis DM, Jones R: Parametric bootstrapping in molecular phylogenetics: applications and performance. In Molecular zoology: advances, strategies and protocols Edited by: Ferraris JD, Palumbi SR. New York: John Wiley and Sons, Inc; 1996:19-45.

52. Yang Z: PAML: a program package for phylogenetic analysis by maximum likelihood. Comput Appl Biosci 1997, I3:555-556.

53. McCluskey J, Gibbs HA, Davies RL: Variation in outer-membrane protein and lipopolysaccharide profiles of Pasteurella haemolytica isolates of serotypes AI and A2 obtained from pneumonic and healthy cattle. Microbiology I994, I40(Pt 4):807-814 